\title{
Microvascular Obstruction Complicating Acute Right Ventricular
}

\section{Myocardial Infarction}

\author{
Biventricular MO
}

${ }^{1}$ Daniele Andreini, MD, ${ }^{1}$ Gianluca Pontone, MD, ${ }^{1}$ Saima Mushtaq, MD, ${ }^{2}$ Jan Bogaert, MD

${ }^{1}$ Mauro Pepi, MD, ${ }^{1}$ Erika Bertella, and ${ }^{3}$ Pier Giorgio Masci, MD.

${ }^{1}$ Centro Cardiologico Monzino, IRCCS, Milan, Italy

${ }^{2}$ Radiology Department - University Hospitals Leuven, Leuven, Belgium

${ }^{3}$ Fondazione C.N.R/Regione Toscana 'G. Monasterio', Pisa, Italy.

Address for correspondence: Daniele Andreini, MD

Via C. Parea 4

20138 Milan

Tel: +39-02-58002577

Tel: +39-02-58002231

E-mail: daniele.andreini@ccfm.it 


\section{ABSTRACT}

We presented a case of a 53-year-old hypertensive man, admitted to the emergency department of our Institute with a diagnosis of inferior ST-segment elevation myocardial infarction (MI) with right ventricular $(\mathrm{RV})$ involvement, treated by percutaneous coronary intervention (PCI) with bare metal stent deployment in the proximally occluded right coronary artery. Post-procedural flow was suboptimal and an ECG at 1-hour after PCI showed incomplete ST-segment elevation resolution. Four days after the acute event, the patient underwent contrast-enhanced cardiovascular magnetic resonance that showed diffuse pericardial effusion, akinetic left ventricular (LV) inferior and inferolateral walls and akinetic right ventricular inferior free-wall with an impairment of global systolic function. Late gadolinium enhancement (LGE) imaging showed transmural LGE of LV inferior and inferolateral walls with extensive microvascular obstruction. Additionally LGE of RV inferior free-wall was also seen with evident hypointense core comprised within hyperenhanced myocardium, consistent with microvascular obstruction of the infarcted RV myocardium. At fourmonth follow-up cine imaging showed persistent akinesia of LV inferior and inferolateral walls and persistent RV global systolic dysfunction. Moreover, LGE of LV inferior and inferolateral walls and RV inferior free-wall was still evident with resolution of microvascular obstruction. This is a peculiar case of a patient with high ischemic and ischemia/reperfusion burden causing extensive damage of coronary microvasculature complicating not only the left but also right ventricular infarcted myocardium.

\section{KEYWORDS}

Acute myocardial infarction; right ventricular infarction; late gadolinium enhancement; microvascular obstruction. 


\section{BACKGROUND}

The clinical and prognostic role of myocardial infarction location and size evaluated by cardiac magnetic resonance (CMR) in patients with acute myocardial infarction (MI) has been recently described (1). The presence of microvascular obstruction (MO) within infarcted region may adversely influence left ventricular remodelling after MI (2).

\section{CASE PRESENTATION}

A 53-year-old man, with history of hypertension and smoking habit, was admitted to the emergency department of our Institute because of acute chest pain. At admission, 7 hours after symptoms onset, the patient was still symptomatic, the physical examination was unremarkable (Killip class I) and blood pressure was 100/70 mmHg. Twelve-lead ECG findings were consistent with acute inferior ST-segment elevation MI with right ventricular (RV) involvement (Figure 1, Panel A). Invasive coronary angiography showed proximally occluded right coronary artery and the patient underwent percutaneous coronary intervention (PCI) with bare metal stent deployment though post-procedural flow was sub-optimal (TIMI flow 2) (Figure 1, Panel C and D). After vessel recanalization he experienced an episode of ventricular fibrillation effectively treated with a single DC shock. Twelve-lead ECG at 1-hour after PCI showed incomplete ST-segment elevation resolution $(<50 \%)$ (Figure 1, panel B). Four days after the acute event, the patient underwent contrast-enhanced CMR (Discovery 450, GE Healthcare, Milwaukee, WI, USA, 1,5 T unit). Cine imaging by steady-state free-precession sequence showed diffuse pericardial effusion and akinetic left ventricular (LV) inferior and inferolateral walls. Right ventricular inferior free-wall was also akinetic yielding an impairment of global systolic function (ejection-fraction=40\%) (Movie A). Ten minutes after the administration of $0.1 \mathrm{mmol} / \mathrm{Kg}$ of Gadolinium-BOPTA (Multihance, Bracco, Milan, Italy), late gadolinium enhancement (LGE) imaging by segmented T1-weighted gradientecho inversion-recovery sequence showed transmural LGE of LV inferior and inferolateral walls with extensive MO. Additionally LGE of RV inferior free-wall was also seen with evident hypointense core comprised within hyperenhanced myocardium (Figure 2, Panel A and B). This 
finding was consistent with MO of the infarcted RV myocardium. At four-month follow-up cine imaging showed resolution of pericardial effusion, persistent akinesia of LV inferior and inferolateral walls and slight functional improvement limited to the basal portion of RV inferior free-wall without any consistent recovery of RV global systolic function (ejection-fraction=43\%) (Movie B). On post-contrast imaging, LGE of LV inferior and inferolateral walls and RV inferior free-wall was still evident with resolution of MO (Figure 2, panel C and D).

To the best of our knowledge this is the first case showing MO complicating infarcted RV freewall. Experimental studies indicate that the right ventricle is more resistant to ischemia than the left ventricle because of its more favorable oxygen demand/supply profile (3). Accordingly it is conceivable that the right ventricle is less predispose to develop ischemia/reperfusion injury at the moment of flow restoration. Microvascular obstruction is a hallmark of ischemia/reperfusion injury, and impedes adequate reperfusion of the previously ischemic myocardium even so the effective recanalization of the infarct-related artery by thrombolysis or primary PCI. Using contrast-enhanced CMR, MO of infarcted LV myocardium has been reported in almost half of the patients with acute ST-segment elevation MI treated by primary PCI (4). On the contrary, MO of infarcted RV freewall has never been detected previously. In a recent study investigating the RV ischemic pattern in a large cohort of acute ST-segment elevation MI patients treated by primary PCI we did not detect RV MO in any of the patients showing LGE of RV free-wall on early post-infarction contrastenhanced CMR (5). However, in the current manuscript we describe a peculiar case of a patient with high ischemic and ischemia/reperfusion burden causing extensive damage of coronary microvasculature complicating not only the left but also right ventricular infarcted myocardium.

\section{CONCLUSIONS}

CMR allowed to detect a very unusual case of left and right ventricular infarction complicated by an extensive damage of coronary microvascular circulation. 


\section{CONSENT}

Written informed consent was obtained from the patient for publication of this Case report and any accompanying images and movies. A copy of the written consent is available for review by the Editor-in-Chief of this Journal.

\section{LIST OF ABBREVIATIONS}

$\mathrm{CMR}=$ cardiac magnetic resonance.

LGE=late gadolinium enhancement.

$\mathrm{LV}=$ left ventricular.

$\mathrm{MI}=$ myocardial infarction.

$\mathrm{MO}=$ microvascular obstruction .

PCI=percutaneous coronary intervention.

$\mathrm{RV}=$ right ventricular.

\section{COMPETING INTERESTS}

The authors declare that they have no competing interests.

\section{AUTHORS' CONTRIBUTIONS}

Daniele Andreini, Saima Mushtaq and Erika Bertella made analysis and interpretation of CMR data. Daniele Andreini and Pier Giorgio Masci have been involved in drafting the manuscript.

Gianluca Pontone, Mauro Pepi and Jan Bogaert have revised the manuscript and given the final approval. 


\section{REFERENCES}

1) Masci PG, Ganame J, Francone M, Desmet W, Lorenzoni V, Iacucci I, Barison A, Carbone I, Lombardi M, Agati L, Janssens S, Bogaert. Relationship between location and size of myocardial infarction and their reciprocal influences on post-infarction left ventricular remodelling. J. Eur Heart J. 2011, 32:1640-8.

2) Bernhard L. Gerber, Carlos E. Rochitte, Jacques A. Melin, Elliot R. McVeigh, David A. Bluemke, Katherine C. Wu, Lewis C. Becker, Joa o A.C. Lima. Microvascular obstruction and left ventricular remodeling early after acute myocardial infarction. Circulation 2000, 101:2734-2741.

3) Bowers TR, O'Neill WW, Grines C, Pica MC, Safian RD, Goldstein JA. Effect of reperfusion on biventricular function and survival after right ventricular infarction. $N$ Engl J Med. 1998, 338:933-940.

4) Hombach V, Grebe O, Merkle N, Waldenmaier S, Höher M, Kochs M, Wöhrle J, Kestler HA. Sequelae of acute myocardial infarction regarding cardiac structure and function and their prognostic significance as assessed by magnetic resonance imaging. Eur Heart J. 2005, 26:549-57.

5) Masci PG, Francone M, Desmet W, Ganame J, Todiere G, Donato R, Siciliano V, Carbone I, Mangia M, Strata E, Catalano C, Lombardi M, Agati L, Janssens S, Bogaert J. Right ventricular ischemic injury in patients with acute ST-segment elevation myocardial infarction: characterization with cardiovascular magnetic resonance. Circulation. 2010, 122:1405-12. 


\section{FIGURE LEGENDS}

\section{Figure 1. ECG and coronary angiography before and after PCI.}

Panel A. Twelve and right ventricular precordial leads before primary PCI showing ST-segment elevation, Q-wave and diphasic T-wave in the inferior leads. In addition, ST-segment elevation is also evident in the right precordial leads (from V3R to V5R)

Panel B. Twelve-lead ECG 1-hour after primary PCI shows incomplete $(<50 \%)$ ST-segment elevation resolution in the inferior leads.

Panel C. Right coronary angiogram before primary PCI showing the occlusion of the proximal portion of the vessel.

Panel D. Right coronary angiogram after primary PCI shows sub-optimal reflow in the vessel (TIMI flow 2).

Figure 2. Cardiac magnetic resonance at 4 days and 4 months after myocardial infarction.

Mid short-axis LGE images at 4 days (panels A and B) and 4 months (panels C and D) after acute MI. Early post- infarction LGE images (panels A and B) show transmural LGE of LV inferior and inferolateral walls with extensive MO. In addition, LGE of RV inferior free-wall is also seen with hypointense core (arrows) comprised within hyperenhanced myocardium. This finding is consistent with MO complicating the infarcted RV myocardium. At 4 months follow-up (panels $\mathrm{C}$ and D), LGE of LV inferior and inferolateral walls and RV inferior free-wall is still evident with resolution of MO.

$\mathrm{MI}=$ myocardial infarction; LGE=late-gadolinium enhancement; $\mathrm{MO}=$ microvascular obstruction; PCI: percutaneous coronary intervention 


\section{MOVIE LEGENDS}

Movie A. Cardiac magnetic resonance, cine short axis imaging at 4 days after myocardial infarction.

Cine short-axis imaging at 4 days after acute MI showing diffuse pericardial effusion, akinesia of LV inferior and inferolateral walls and of RV inferior free-wall.

Movie B. Cardiac magnetic resonance, cine short axis imaging at 4 months after myocardial infarction.

Cine short-axis imaging 4 months after acute MI showing resolution of pericardial effusion but persistent akinesia of LV inferior and inferolateral wall and slight functional improvement limited to the basal portion of RV inferior free-wall. 
A tolindicto

Dim

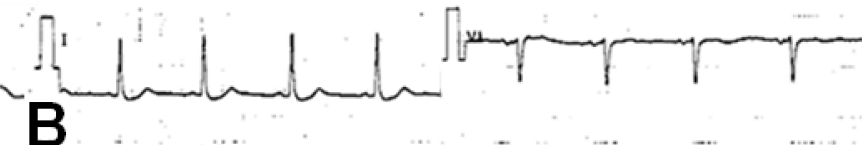
B

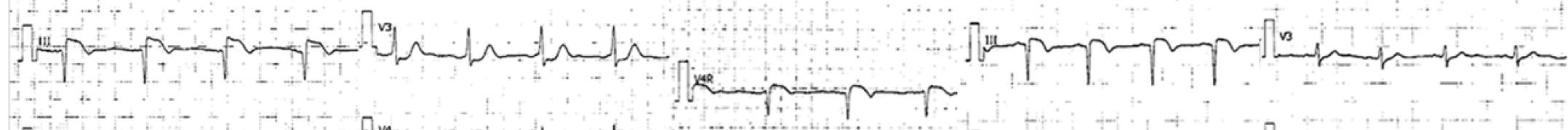

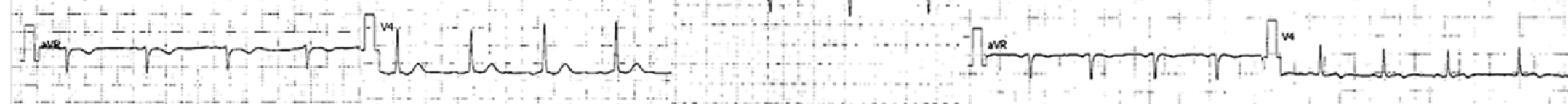

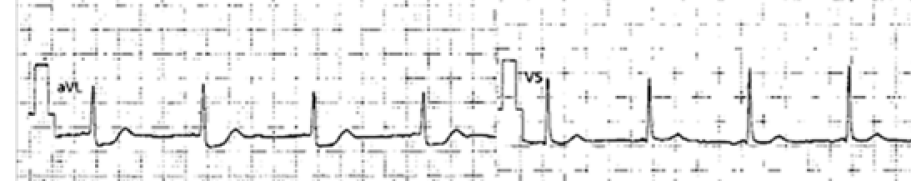

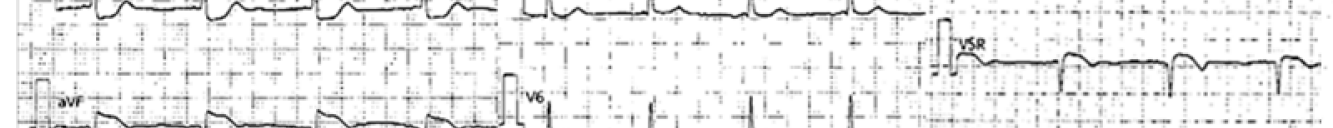

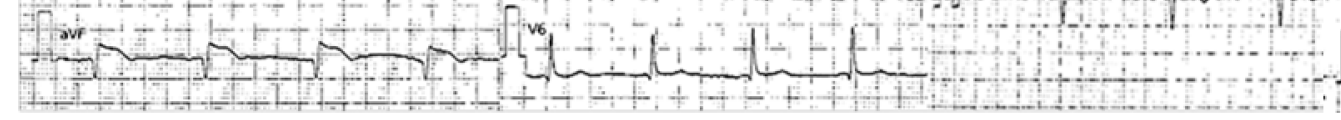

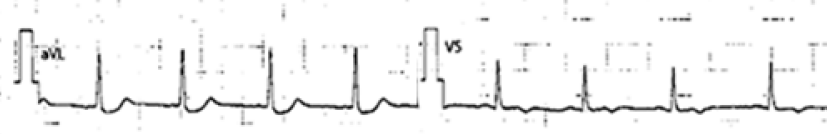

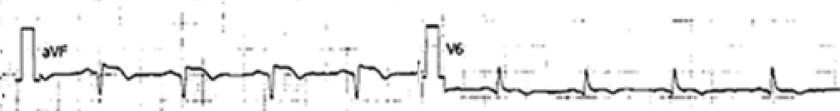
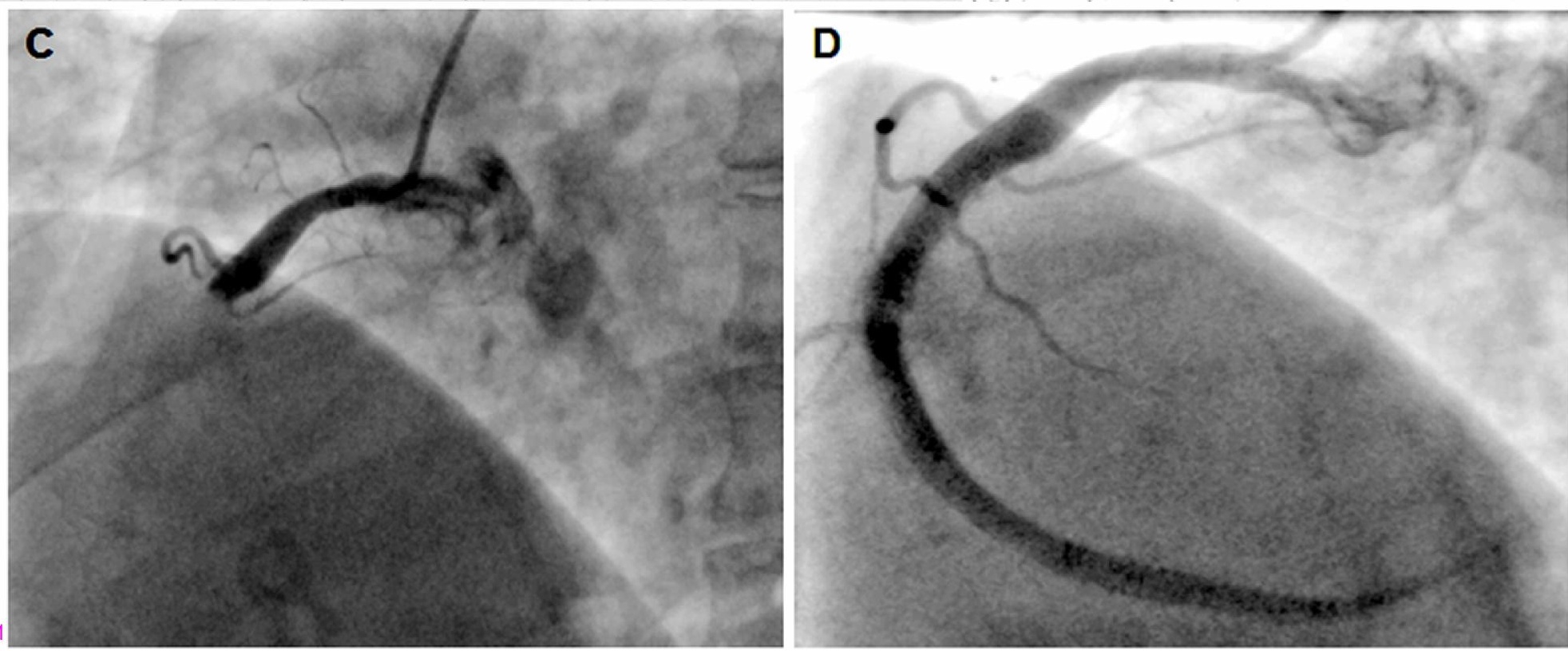
Additional files provided with this submission:

Additional file 1: Movie A.mpg, 10566K

http://jcmr-online.com/imedia/1549214609683475/supp1.mpeg

Additional file 2: Movie B.mpg, 10333K

http://jcmr-online.com/imedia/1745971042683475/supp2.mpeg 